\title{
Heteroflocculation of Amidine Polystyrene Latex and Anticarsia gemmatalis Nucleopolyhedrovirus as a Model System for Studying Sunlight Protection
}

\author{
Marina de Moraes Lessa and Claudia Conti Medugno ${ }^{1}$ \\ Embrapa Environment, C.P. 69, CEP 13820-000 Jaguariúna, Brazil
}

Received July 19, 1999; accepted January 28, 2000

\begin{abstract}
Anticarsia gemmatalis nucleopolyhedrovirus(AgM NPV) isa baculovirus specific for the control of an important soybean defoliator. The baculovirus is comprised of double-stranded D NA, occluded in a proteinaceous structure called a polyhedron. U Itraviolet sunlight is the most destructive factor that affects the persistence of the virus in the field. In the present study, we use a model system wherein the pathogen is covered by another particle of opposite charge in order to test the effectiveness of a physical barrier as a protection against sunlight. $H$ eteroflocculation experiments were carried out using two different age batches of AgMNPV and amidine polystyrene latex particles. Theassessment of heter oflocculation was achieved by zeta potential and adsorption isotherm measurements, and by scanning electron microscopy. Despite the great difference in potentials between latex particles and the baculovirus, low-affinity isotherms were obtained in both pure water and $0.1 \mathrm{mM} \mathrm{K} \mathrm{Cl}$. Adsorbed latex particles were easily washed out from the polyhedron surface. This low affinity could be attributed to the presence of a strongly repulsive hydration force of short range operating on the system. The results suggest that the failure to obtain a good physical barrier against sunlight might be attributed to the difficulty in keeping the polyhedron surface covered.

Key Words: baculovirus; AgMNPV; ultraviolet; heteroflocculation; hydration forces.
\end{abstract}

\section{INTRODUCTION}

Baculoviruses (BV) are highly specific and often virulent viruses that infect insects mainly of the lepidopteran (caterpillar) species. Anticarsia gemmatalis nucleopolyhedrovirus (AgMNPV) is a baculovirus specific for the control of velvetbean caterpillar A. gemmatalis, which is one of the main soybean defoliators in a number of countries. The use of AgMNPV in the soybean fields of Brazil first began in 1979 and represents one of the most successful biological control programs in the world (1).

Despite this success, commercial formulations do not possess a good persistence under field conditions, with the virus being in-

\footnotetext{
${ }^{1}$ To whom correspondence should be addressed. Fax: (055) 0198678740 or 8678799. E-mail: medugno@cnpma.embrapa.br.
}

activated by exposure to sunlight. Several attempts to overcome this drawback have been made and include the use of encapsulation or physical barriers such as clays or carbon (2), UV absorbers $(2,3)$, and brighteners (4). Despite all of these efforts, the study of the interaction between the protectors and the pathogen surface, which is the first step in a successful formulation, has been neglected, and therefore only a few examples can be found in the current literature $(5,6)$. In order to test the effectiveness of the use of a physical barrier as a sunlight screen, a model system in which the pathogen is covered by another particle of an elevated refractive index is proposed. The coating of polyhedra can be achieved by allowing the particle to interact with another of an opposite surface charge. AgMNPV is comprised of double-stranded DNA that is occluded in a proteinaceous structure called a polyhedron, which is surrounded by an envelope of densely stained material, the principal component of which is a carbohydrate-associated protein $(7,8)$. Studies on the surface of AgMNPV have shown that this particle of $1.3 \pm 0.2 \mu \mathrm{m}$ has a negative surface charge when the ambient $\mathrm{pH}$ is higher than 4 (9). Latex polystyrene particles were chosen to coat BV because they are monodisperse and can be produced or purchased in different sizes and with different superficial properties.

Interactions between colloidal particles are often explained by DLVO theory $(10,11)$ where attractive van der Waals and repulsive double-layer forces play a central role. This theory has been extended to the case of heteroflocculation $(12,13)$, and has also been experimentally tested $(14,15)$. However, many colloidal systems can be fully understood only by considering other types of interactions (16). For instance, biological surfaces and macromolecules remain separated in aqueous solutions at high ionic strengths (17-19), a condition under which coagulation is predicted to occur by DLVO. The explanation for this is that the molecules experience an extra repulsive force at high ionic strengths. The large repulsive forces occurring between surfaces separated by a distance of $1 \mathrm{~mm}$ and in water have been measured using surface force apparatus $(19,20)$. As this force occurs in solids of low energy of wetting with water, the repulsive force can be attributed to the energy required to remove the water of hydration from the surface. These non-DLVO forces are known as structural or hydration forces. In this paper we investigate the 
heteroflocculation of the baculovirus and amidine polystyrene latex in water, the heteroflocculation being carried out using two batches of the baculovirus and three samples of latex amidine polystyrene of different sizes.

\section{MATERIAL AND METHODS}

\section{AgMNPV Purification}

Fifth-instar larvae of A. gemmatalis reared on an artificial diet at $28^{\circ} \mathrm{C}$ were infected with a suspension of $10^{7}$ polyhedra $/ \mathrm{ml}$. Six to ten days after infection, the larvae exhibiting symptoms of nucleopolyhedrosis were frozen at $-18^{\circ} \mathrm{C}$. The polyhedra were then purified using a modification of the method proposed by van der Geest (21). After defrosting, the larvae were ground, filtered through a synthetic fabric, and diluted to a concentration of $10 \mathrm{~g} \mathrm{dm}^{-3}$ of solids in $1 \%$ sodium dodecyl sulfate (SDS). The suspension was then centrifuged at $5000 \mathrm{~g}$ and the solids were resuspended in water. The operation was repeated until polyhedra of a high enough degree of purity could be observed by an optical microscope; the concentrated dispersions were then kept at $4{ }^{\circ} \mathrm{C}$. Two batches of the baculovirus were used in the present work: a freshly prepared dispersion, BV-1, and a 6-month-old preparation, BV-2; both batches exhibited the same biological activity as measured according to Ref. (22) and the time delay between experiments was not greater than 1 month.

\section{Amidine Polystyrene Latex}

Latex was purchased from the Interfacial Dynamic Corp. and prepared by diluting the supplied stock to the appropriate particle concentration $\left(n_{\text {add }} / \mathrm{ml}\right)$; the properties of the latex used are given in Table 1. Distilled water purified in a deionizer was used for all particle dispersions.

\section{Particle Size Distribution and Zeta Potential}

The particle size distribution and zeta potential were measured using Zetaplus equipment from Brookhaven Instruments. The zeta potential was measured using a laser-doppler operating system containing a laser source of $670 \mathrm{~nm}$, at a sampling time of $256 \mu$ s and a modulation frequency of $250 \mathrm{~Hz}$; the reported potential represents the result of averaging five consecutive runs. The procedure was as follows. First, the potentials of latex dispersions of different concentrations were determined in order to establish the optimum measurement range and the minimum

TABLE 1

\section{Properties of the Latex U sed To C oat the Baculovirus (from Certificate of Supplier)}

\begin{tabular}{ccc}
\hline Size $(\mu \mathrm{m})$ & $\begin{array}{c}\text { Surface charge density } \\
\left(\mu \mathrm{C} / \mathrm{cm}^{2}\right)\end{array}$ & $\begin{array}{c}\text { Area per charge group } \\
\left(A^{2} / C\right)(\mathrm{NH}) \mathrm{NH}_{2}\end{array}$ \\
\hline $0.12 \pm 14 \%$ & 8.1 & 198 \\
$0.172 \pm 2.3 \%$ & 2.24 & 715 \\
$0.954 \pm 4.3 \%$ & 16.12 & 99 \\
\hline
\end{tabular}

concentration the instrument was able to detect. The same procedure was adopted for all baculovirus dispersions. Second, latex particles were added to $10 \mathrm{ml}$ of baculovirus suspensions containing $(1.1 \pm 0.2) \times 10^{6}$ polyhedra $/ \mathrm{ml}$. The concentration of the particles was calculated for a given ratio between the numbers of particles and polyhedra. The concentration of latex particles was kept lower than the detection limit to ensure that the measured value represented only bare or covered baculovirus mobility. The mixed particles were allowed to interact in a test tube, and then transferred to an appropriate cell and the electrophoretic mobility was then determined. Results are expressed as the potential of BV against the ratio between the added particles and $\mathrm{BV}$.

\section{Scanning Electron Microscopy (SEM)}

Morphological studies were performed using photomicrographs obtained from a scanning electronic microscope, LEO Estereoscan 440. The samples were metalized with gold using Bal-Tec MED 020 Sputter Coat devices. The samples were obtained by contact dispersions of $5.0 \mathrm{ml}$ of $1.3 \times 10^{8}$ polyhedra $(\mathrm{BV}-1) / \mathrm{ml}$ and $2.5 \mathrm{ml}$ of $6.8 \times 10^{11}$ latex particles $/ \mathrm{ml}$, each being prepared by carefully drying a suspension of the heteroaggregates over a carbon double face and then fixed in the aluminum sample support.

\section{Adsorption Experiments}

Heteroflocculation was achieved by mixing suspensions of the baculovirus and latex particles of an appropriate concentration at $25^{\circ} \mathrm{C}$ and allowing the interaction to take place with mild agitation in a centrifuge tube. The mixtures were then centrifuged for $30 \mathrm{~min}$ at $2000 \mathrm{rpm}(400 \mathrm{~g})$. Control experiments showed that the absorbance of latex suspensions does not change before or after centrifugation under the above conditions and that zero absorbance can be measured for the supernatant of the centrifuged baculovirus (BV-1 or BV-2) suspensions. As free latex does not sediment, the number of particles not bound to the baculovirus can be determined by turbidity measurements by carefully withdrawing the supernatant to a spectrophotometer cell and comparing the absorbance at $400 \mathrm{~nm}$ with the latex calibration curve.

\section{RESULTS}

\section{Electrophoretic Experiments}

The zeta potentials of all of the particles used in this paper are given in Table 2. Figure 1 displays the zeta potential of baculovirus dispersions as a function of the ratio $n_{\mathrm{add}} / n_{\mathrm{BV}}$, where $n_{\text {add }}$ is the number of latex particles added and $n_{\mathrm{BV}}$ is the number of polyhedra. It can be seen that the potential slowly increases to zero and then reaches a plateau. For $0.12-\mu \mathrm{m}$ latex particles, the number of particles required to reach the isoelectric point depends on the age of the baculovirus preparation. For a 6-month-old preparation (BV-2) $3300 \pm 300$ particles/ 
TABLE 2

Zeta Potential and Detection Limit for Polyhedra and Latices in Laser-D oppler Experiments

\begin{tabular}{lcc}
\hline \multicolumn{1}{c}{ Particle } & $\begin{array}{c}\text { Zeta potential } \\
(\mathrm{mV})\end{array}$ & $\begin{array}{c}\text { Minimum concentration } \\
\text { detected (number of } \\
\text { particles per ml) }\end{array}$ \\
\hline BV-1 & $-33 \pm 2$ & $1 \times 10^{4}$ \\
BV-2 $(0.120 \mu \mathrm{m})$ & $-33 \pm 2$ & $1 \times 10^{4}$ \\
Latex & $44 \pm 1$ & $1 \times 10^{10}$ \\
Latex $(0.954 \mu \mathrm{m})$ & $21 \pm 3$ & $1 \times 10^{5}$ \\
\hline
\end{tabular}

polyhedron were found to be required compared to only 1200 particles/polyhedron for the fresh preparations (BV-1). The same titration profile was observed for $0.954-\mu \mathrm{m}$ latex particles, where 17 particles/polyhedron (BV-2) were found to be necessary in order to reach a zero zeta potential.

\section{Adsorption Isotherms}

In Fig. 2, the adsorption curves for latex particles of $0.120-\mu \mathrm{m}$ diameter are shown for both the $\mathrm{BV}-1$ and $\mathrm{BV}-2$ preparations (Figs. $2 \mathrm{a}$ and $2 \mathrm{~b}$, respectively) and $0.172-\mu \mathrm{m}$ latex particles and BV-1 (Fig. 2c). In Figs. 2a and $2 b$ the upper axis shows the ratio $n_{\text {add }} / n_{\mathrm{BV}}$, which represents the number of $0.120-\mu \mathrm{m}$ latex particles added per polyhedron, thus allowing for a comparison between the zeta potential curves and adsorption measurements. Flocculation can be observed for BV-1 with $0.120-\mu \mathrm{m}$ latex particles when the ratio between the numbers of added latex particles and $\mathrm{BV}\left(n_{\mathrm{add}} / n_{\mathrm{BV}}\right)$ is in the range $1000-1400$ (Fig. 2a). One can see in Fig. 1A that this is the relationship $n_{\text {add }} / n_{\mathrm{BV}}$ where zero zeta is reached. Figure 3 shows the number of $0.120-\mu \mathrm{m}$ latex particles adsorbed per polyhedron as a function of the relationship between the numbers of added latex particles and BV-1, in $0.1 \mathrm{mM} \mathrm{KCl}$ and in water. All curves display an "S"-type behavior that can be identified as a low-affinity
TABLE 3

Covering F actor for Latex Particles over the Baculovirus

\begin{tabular}{cccc}
\hline $\begin{array}{c}\text { Latex } \\
\text { diameter }(\mu \mathrm{m})\end{array}$ & $\begin{array}{c}\text { Maximum number } \\
\text { of latices per } \\
\text { polyhedron }\end{array}$ & $\begin{array}{c}\text { Number of latices } \\
\text { per polyhedron } \\
\text { from adsorption } \\
\text { isotherms (plateau) }\end{array}$ & $\begin{array}{c}\text { Covering } \\
\text { factor }\end{array}$ \\
\hline 0.120 & 776 & $550^{a}$ & 0.64 \\
0.172 & 329 & 187 & 0.568 \\
0.954 & 6.5 & Not determined & Not determined \\
\hline
\end{tabular}

${ }^{a} \mathrm{BV}-1$ and $\mathrm{BV}-2$.

isotherm (23). For $0.120-\mu \mathrm{m}$ latex particles, a plateau is reached at $550 \pm 50$ latex particles/polyhedron (BV-1); in older preparations (BV-2), no such plateau can be clearly identified. The adsorption of latex rises with an increasing number of added particles and a small decrease in the maximum number of particles adsorbed per BV-1 can be observed when using $0.1 \mathrm{mM}$ $\mathrm{KCl}$. For $0.172-\mu \mathrm{m}$ latex particles, the plateau can be observed at 188 latex particle per BV-1 polyhedron. Visual observations of the two BV preparations show that in the BV-2 samples, a small number of polyhedra are able to float and remain at the air-water interface. Those particles were removed before the adsorption experiments, but were observed again after $24 \mathrm{~h}$.

The ratio between the area of a baculovirus and the area of a square with a side of dimension equal to the latex particle diameter can be assumed as being approximately the maximum number of latex spheres that could cover the entire polyhedron surface. The relationship between the number of particles adsorbed per baculovirus at the plateau and this maximum number of particles is taken as the covering factor. Table 3 shows the covering factor for the two particle sizes examined. Covering factors, as defined in this paper (Table 3), are in accordance with the expected trend; that is, smaller particles cover a bigger fraction of the polyhedron surface.
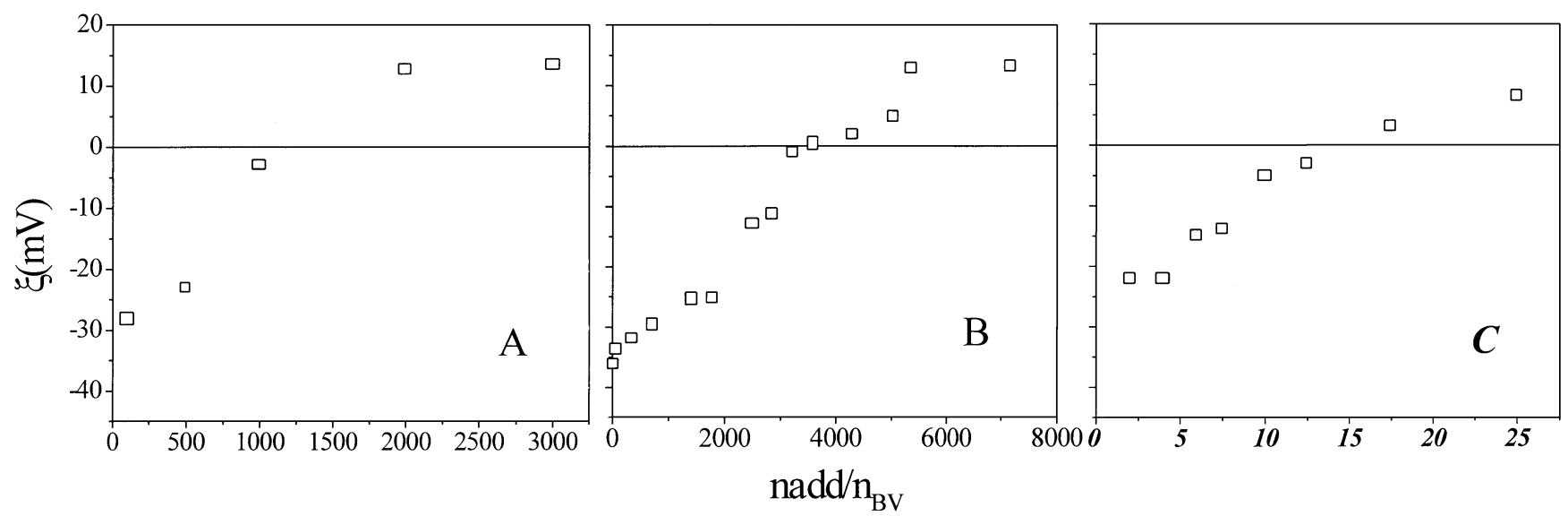

FIG. 1. Zeta potential of the baculovirus as a function of $n_{\mathrm{add}} / n_{\mathrm{BV}}$, the ratio between the number of added latex particles and polyhedra at $25^{\circ} \mathrm{C}$. Curve $\mathrm{A}$, $0.120-\mu \mathrm{m}$ latex and BV-1; curve B, 0.120- $\mu \mathrm{m}$ latex and BV-2; curve C, $0.954-\mu \mathrm{m}$ latex and BV-1. Concentration of $\mathrm{BV}$ in all curves is $3.4 \times 10^{6}$ polyhedra/ml. 

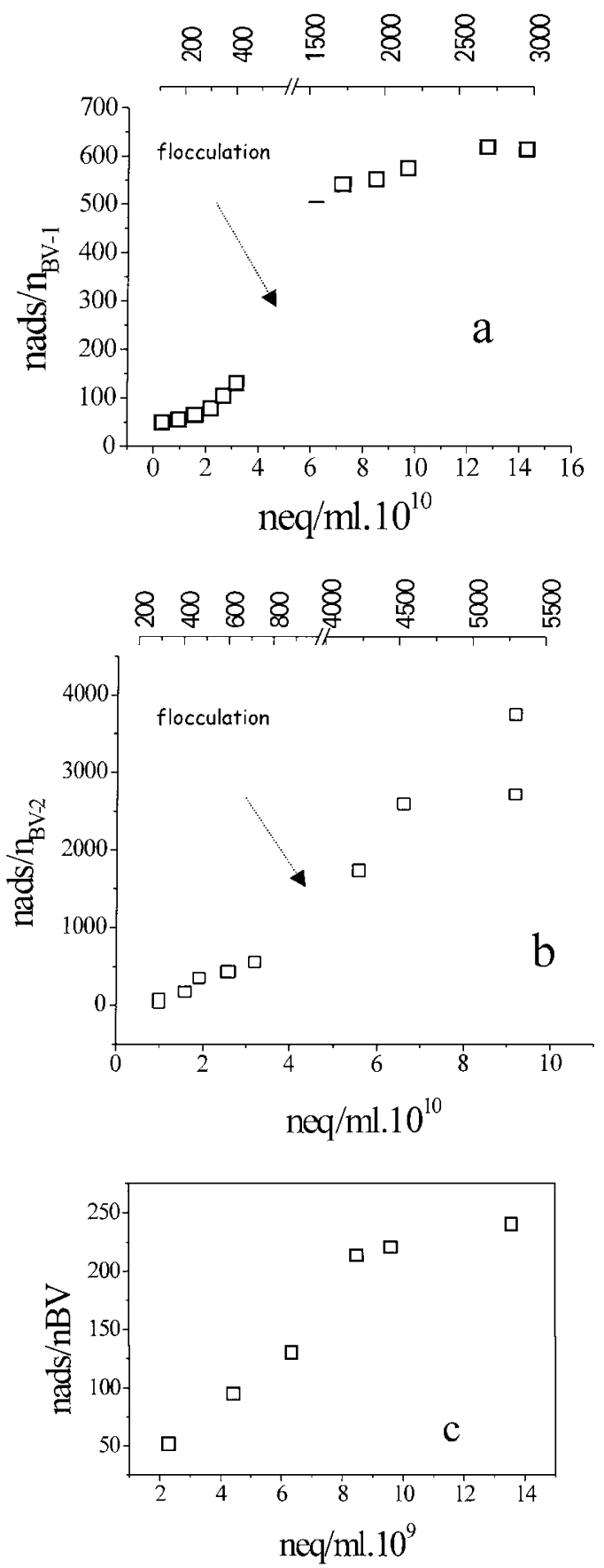

FIG. 2. Adsorption isotherms of latex particles on polyhedra. Curve a, $0.120-\mu \mathrm{m}$ latex and BV-1; curve b, 0.120- $\mu \mathrm{m}$ latex and BV-2; curve c, $0.172-\mu \mathrm{m}$ latex and $\mathrm{BV}-1 . n_{\mathrm{ads}} / n_{\mathrm{BV}}$ is the number of latex particles absorbed per BV-1; $n_{\text {add }} / n_{\mathrm{BV}}$ is the number latex particles added per $\mathrm{BV} ; n_{\mathrm{eq}} / \mathrm{ml}$ is the number of latex particles in equilibrium. Concentration of BV-1 is $3.4 \times 10^{6}$ polyhedra $/ \mathrm{ml}$. Upper axis shows the ratio between the number of added latex particles and $\mathrm{BV}$ $\left(n_{\mathrm{add}} / n_{\mathrm{BV}}\right)$.

\section{Morphological Characterization}

SEM of two samples of the baculovirus coated with $0.120-\mu \mathrm{m}$ latex particles are shown in Fig. 4, where the heteroaggregates

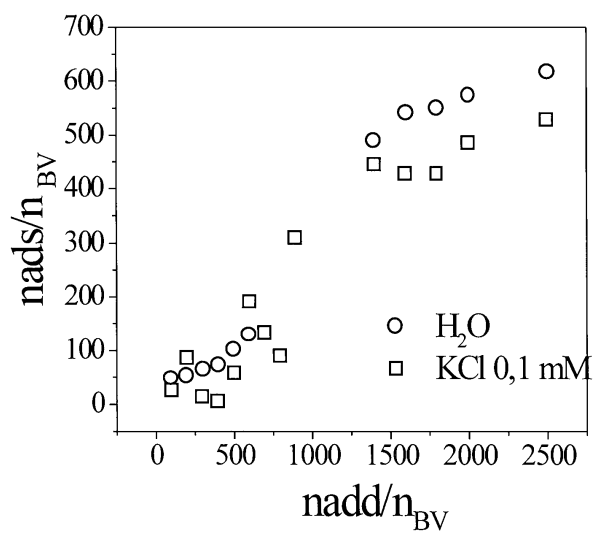

FIG. 3. Adsorption curves of $0.120-\mu \mathrm{m}$ latex particles onto the polyhedron surface. $n_{\text {ads }} / n_{\mathrm{BV}}$ is the number of latex particles absorbed per BV-1 and $n_{\text {add }} / n_{\mathrm{BV}-1}$ is the number of $0.120-\mu \mathrm{m}$ latex particles added per BV-1. Concentration of $\mathrm{BV}-1$ is $3.4 \times 10^{6}$ polyhedra/ml. Circles represent experiments carried out in water; squares represent experiments carried out in $0.1 \mathrm{mM} \mathrm{KCl}$.

can easily be seen, as well as the polyhedra surfaces with bare regions.

\section{DISCUSSION}

According to DLVO, when two particles of opposite surface charge are allowed to interact, flocculation should occur (10-13). The remarkable fact about the heteroflocculation of the baculovirus and amidine latex particles is that, despite the great zeta potential difference between these two particles, lowaffinity isotherms ("S"-shaped curves) can be obtained (Fig. 2), and bare regions on the polyhedron surface can be observed on SEM (Fig. 4). This behavior was unexpected; attraction between latex particles and the baculovirus should be very strong, and aggregation therefore irreversible.

Different possibilities can be considered to explain this anomalous behavior. The first is lateral repulsion between adsorbed latices. In considering adsorption of oppositely charged particles, two sets of electrostatic interactions must be taken into account: the attractive interactions normal to the interface, i.e., the primary adsorption forces, and the interactions parallel to the interface, i.e., the lateral repulsive interactions between adsorbed particles. The lateral interaction was found to be responsible for the low-affinity isotherms at high electrolyte concentration reported in the adsorption of small positive latices onto negative polystyrene latex particles, both of which had been sterically stabilized (24). In this system, a high-affinity isotherm was obtained at a low electrolyte concentration. On increasing the salt concentration, the electrostatic adsorbing force and the lateral repulsion force were found to simultaneously decrease and therefore a low-affinity isotherm was obtained (24). As lateral repulsion was diminished, an increase in the covering factor was also observed. In the present case, a low affinity was observed even in water and a small salt effect was observed upon addition of $0.1 \mathrm{mM} \mathrm{KCl}$. No increase in covering factor was observed 


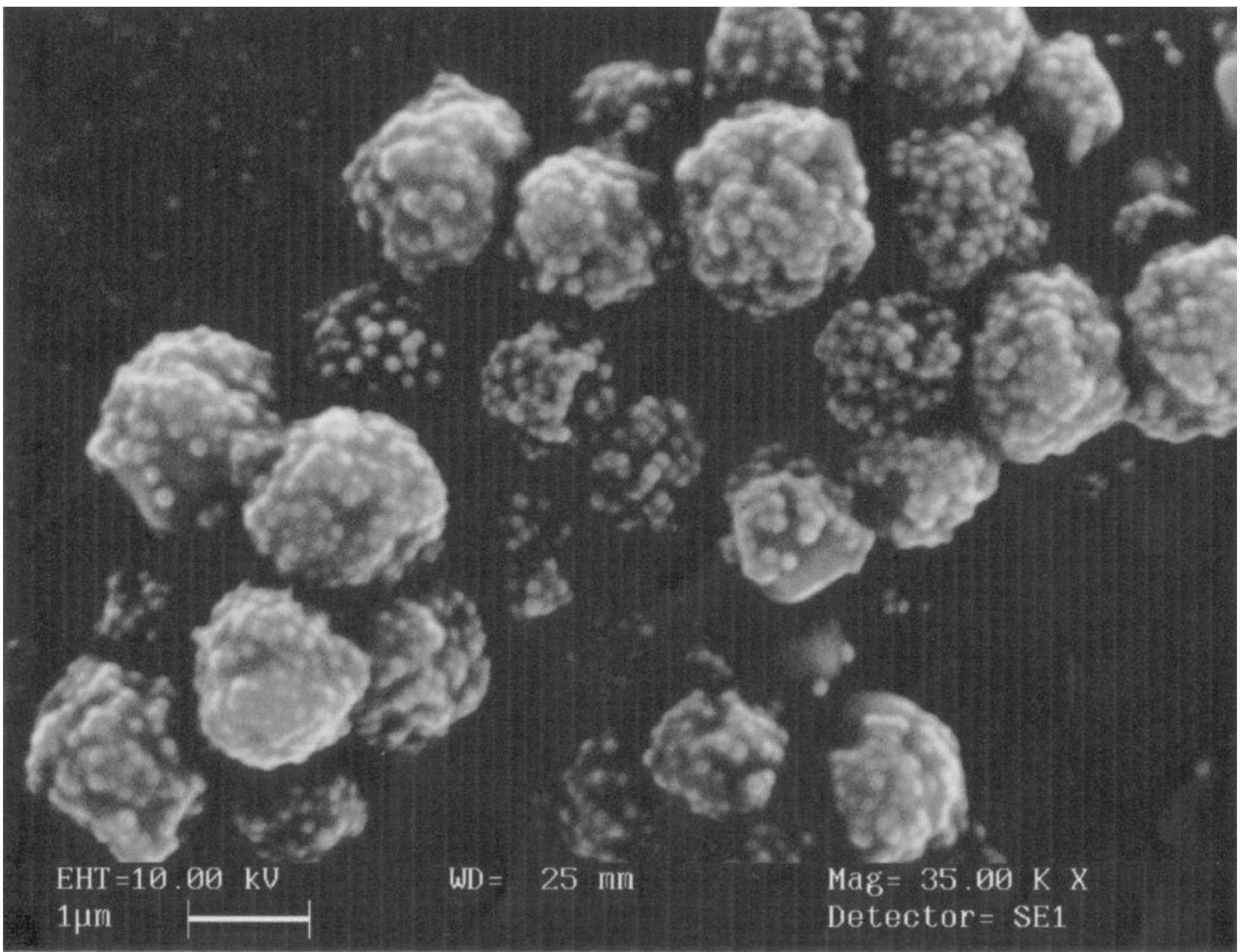

FIG. 4. SEM of $0.120-\mu \mathrm{m}$ latex adsorbed on BV-1. Heteroflocculates are obtained when the relationship between the numbers of polyhedra and latices is equal to the isoelectric point.

upon addition of salt (Fig. 3). The number of adsorbed particles per BV-1 remained essentially constant. Lateral repulsion cannot be responsible for the low affinity observed.

The second possibility is the presence of an extra repulsive force that is able to overcome the expected electrostatic attraction. Hydration forces are widely recognized as non-DLVO mechanisms that provide extra stabilization in colloidal systems (19). They act at surface separations less than 2-4 $\mathrm{nm}$ and are believed to be due to solvent structural effects. It is well known that protein surfaces are strongly hydrated $(19,20)$. Water molecules surrounding charges or polar groups in the particle surface cause an energetic barrier to a close approximation between particles. The stability of immunolatex formed by protein fragments and copoly(styrene-choromethylstyrene) in high salt concentrations is, for instance, attributed to hydration forces $(17,18)$.

The proteins of the baculovirus envelope are probably able to structure water at the polyhedron surface, keeping away other surfaces, making heteroflocculation or adhesion of oppositely charged particles to the baculovirus surface difficult. Hydration repulsive forces are probably responsible for the failure of most physical barriers introduced by heteroflocculation to protect the baculovirus from sunlight once the protector is not bound to a polyhedron surface but dispersed in water. Biological tests of the effectiveness of a physical barrier are meaningless if the interaction between a baculovirus surface and the protector, as well as the covering factors, is not well established. The floating ability of the polyhedra observed in older preparations can be attributed to a hydrophobic character of the baculovirus surface. The mechanism by which the surface becomes hydrophobic is unknown, but most probably occurs at the water-air interface. The hydrophobic character of the baculovirus has been reported in the literature $(5,25)$ although no reference is made to the relationship between aging and hydrophobicity. These hydrophobic groups could be responsible for a long-range hydrophobic attraction between the covered 6-month-old polyhedra and latices. The observations related to surface modifications with time raise more questions than we can answer at present. However, their technological relevance requires further experiments to test the hypotheses raised in the present discussion. Attempts to obtain a good model system wherein small particles are irreversibly 
bound to a polyhedron surface by heteroflocculation are currently being carried out in our laboratory, using different latex and experimental conditions.

\section{CONCLUSIONS}

In the work described in this paper we attempted the adsorption of amidine polystyrene latex particles onto an oppositely charged baculovirus. Despite the large difference in the zeta potential between the two particles, the adsorption was found to be reversible and of a low affinity; hydration forces seemed to be responsible for this unexpected behavior. The aging of the baculovirus changes its surface properties without altering the zeta potential, such that 6-month-old preparations are more hydrophobic. Thus, from the viewpoint of coating polyhedra with oppositely charged particles, the experimental conditions under which the hydration forces can be overcome are still to be determined.

\section{ACK NOW LED GMENTS}

We thank Dr. Fernando Galembeck for his helpful discussions and for facilitating the use of the Brookhaven equipment at UNICAMP.

\section{REFERENCES}

1. Moscardi, F., and Sosa-Gomes, R., in "International Crop Science I" (D. R. Buxton, R. Shibes, R. A. Forsberg, B. L. Blad, K. H. Asay, G. M. Paulsen, and R. F. Wilson, Eds.), pp. 15-119. Crop Science Society of America, Madison, WI, 1993.

2. Ignoffo, C. M., Garcia, C., and Saathoff, S. G., Environ. Entomol. 26, 1470 (1997).
3. Ignoffo, C. M., and Garcia, C., J. Invertebr. Pathol. 67, 192 (1996).

4. Dougherty, E. M., Guthrie, K. P., and Shapiro, M., Biol. Contr. 7, 71 (1996).

5. Small, D. A., Moore, F. N., and Entwistle, P. F., Appl. Environ. Microbiol. 52, 220 (1986).

6. Small, D. A., and Moore, N. F., Appl. Environ. Microbiol. 53, 598 (1987).

7. Minion, F. C., Coons, L. B., and Broome, J. R., J. Invertebr. Pathol. 34, 303 (1979).

8. Lee, S.-Y., Poloumienko, A., Belfry, S., Qu, X., Chen, W., MacAfee, N., Morin, B., Lucarotti, C., and Krause, M., Arch. Virol. 141, 1247 (1996).

9. Medugno, C. C., Lessa, M., and Gonzáles, G., J. Dispersion Sci. Technol. 14, 703 (1993).

10. Derjaguin, B. V., and Landau, B. V., Acta Phys. Chim. URSS 14, 633 (1941).

11. Verwey, E. J. W., and Overbeek, J. Th. G., "Theory of Stability of Liophobic Colloids." Elsevier, Amsterdam, 1948.

12. Derjaguin, B. V., Discuss. Faraday Soc. 18, 85 (1954).

13. Devereux, O. F., and De Bruyn, P. L., "Interaction of Plane Parallel Double Layers." MIT Press, Cambridge, MA, 1963.

14. Islam, A. M., Chowdhry, B. Z., and Snowden, M. J., Adv. Colloid Interface Sci. 62, 109 (1995).

15. Overbeek, J. Th. G., J. Colloid Interface Sci. 58, 408 (1997).

16. Cardoso, A. O., and Galembeck, F., J. Colloid Interface Sci. 182, 614 (1996).

17. Molina-Bolívar, J. A., Galisteo-Gonzáles, F., and Hidalgo-Álvarez, R., Colloids Surf. B. Biointerfaces 8, 73 (1996).

18. Molina-Bolívar, J. A., Galisteo-Gonzáles, F., and Hidalgo-Álvarez, R., J. Colloid Interface Sci. 206, 518 (1998).

19. Israelachvili, J. N., Chem. Scr. 25, 7 (1985).

20. Pashley, R. M., and Israelachvili, J. N., J. Colloid Interface Sci. 101, 511 (1984).

21. van der Geest, L. P. S., J. Invertebr. Pathol. 11, 502 (1968).

22. Medugno, C. C., Ferraz, J. M. G., Maia, A. H., and Freitas, C. C. L., Pestic. Sci. 51, 153 (1997).

23. Giles, C. H., MacEwan, T. H., Nakhwa, S. N., and Smith, D., J. Chem. Soc. 3973 (1960).

24. Vincent, B., Young, C. A., and Tadros, T. F., J. Chem. Soc., Faraday Discuss. 65, 296 (1978).

25. Nordin, G. L., and Maddox, J. V., J. Invertebr. Pathol. 18, 316 (1971). 